\title{
Pre-profiling factors influencing serum microRNA levels
}

\author{
Sara A MacLellan ${ }^{1}$, Calum MacAulay ${ }^{1}$, Stephen Lam ${ }^{1}$ and Cathie Garnis ${ }^{1,2^{*}}$
}

\begin{abstract}
Background: MicroRNAs (miRNAs) are non-coding RNAs that negatively regulate gene expression by preventing the translation of specific mRNA transcripts. Recent studies have shown that miRNAs are stably expressed in human serum samples, making them good candidates for the non-invasive detection of disease. However, before circulating miRNAs can be used reliably as biomarkers of disease, the pre-measurement variables that may affect serum miRNA levels must be assessed.

Methods: In this study we used quantitative RT-PCR to examine the effect of hemolysis, fasting, and smoking on the levels of 742 miRNAs in the serum of healthy individuals. We also compared serum miRNA profiles of samples taken from healthy individuals over different time periods to assess normal serum miRNA fluctuations.

Results: We have found that mechanical hemolysis of blood samples can significantly alter serum miRNA quantification and have identified 162 miRNAs that are significantly up-regulated in hemolysed serum samples. Conversely, fasting and smoking were demonstrated to not have a significant effect on the overall serum miRNA profiles of healthy individuals. The serum miRNA profiles of matched samples taken from individuals over varying time periods showed a high correlation and no miRNAs were significantly differentially expressed in these samples further suggesting the utility of serum miRNAs as biomarkers of disease. Taking the above results into consideration, we have identified miR-99a-5p and miR-139-5p as novel endogenous controls for serum miRNA studies due to their consistency across all sample sets.
\end{abstract}

Conclusion: These results identify important pre-profiling factors that should be taken into consideration when identifying endogenous controls and candidate biomarkers for circulating miRNA studies.

Keywords: MicroRNA, Cancer, Biomarker, Serum, miR-99a-5p, miR-139-5p

\section{Background}

MicroRNAs (miRNAs) are small, endogenous, non-coding RNAs that negatively regulate gene expression by interfering with the translation of target mRNAs [1]. Currently, over 1600 mature miRNA sequences have been identified in humans (miRBase, Release 19.0, http://www.mirbase. org). Each miRNA can target multiple mRNAs and it has been estimated that over $30 \%$ of the human genome is regulated by miRNAs [2]. Consequently, miRNAs have been shown to be involved in many cancer-associated cellular processes including development, cell proliferation, apoptosis, fat metabolism, and cell differentiation [3-8].

\footnotetext{
* Correspondence: cgarnis@bccrc.ca

'Department of Integrative Oncology, British Columbia Cancer Research Centre, Vancouver, BC, Canada

${ }^{2}$ Department of Surgery, University of British Columbia, Vancouver, BC, Canada
}

\section{Ciomed Central}

Until recently, miRNAs were thought to be unstable in the extracellular environment but two independent studies published in 2008 confirmed that miRNAs were present in a stable form in clinical samples of serum and plasma $[9,10]$. These groups also demonstrated that circulating miRNAs exhibit altered expression under certain disease states and could potentially be used as diagnostic and/or prognostic biomarkers. Since 2008, a number of studies have identified candidate miRNA biomarkers for numerous conditions including drug-induced liver injury [11], acute myocardial infarction [12], and various cancers [13-16]. However, studies identifying miRNA biomarkers for the same disease have shown variable results. Due to the lack of a full understanding of circulating miRNAs, the factors that may affect their quantification are difficult to predict. Some of this variability may be attributed to 
pre-profiling sources such as patient lifestyle and sample collection methods. Other possible sources of variability in circulating miRNA profiles are analytical differences such as the quantification platform or normalization methods used [17].

Before circulating miRNAs can reliably be used as biomarkers of disease, the pre-profiling factors that may contribute to inconsistent miRNA quantification must be identified and the variability of circulating miRNAs in healthy individuals must be determined. Recent studies have shown that the majority of miRNAs found in serum and plasma are also present in blood cells and that hemolysis during sample collection or processing may affect the miRNA profile of the sample $[10,18,19]$. In particular, circulating miRs 451a, 16-5p, 24-3p, 15b-5p, 223$3 p$, 486-5p, 150-5p and 92a-3p have all been shown to be inconsistently expressed in healthy individuals due to their presence in blood cells [20,21]. Additionally, studies examining a large number of miRNAs in serum and plasma have shown that many of the miRNAs most commonly expressed in these samples show an altered expression in samples exhibiting blood cell contamination [22,23]. Therefore, the effects of mechanical hemolysis on whole micronome serum quantification require further examination in patient-matched samples to ensure that potential biomarkers are not compromised by blood cell contamination.

Furthermore, it has been suggested that lipid intake before blood draw may interfere with miRNA extraction leading to a variable profile [24] and that miRNAs present in food may influence serum miRNA profiles of healthy individuals [25]. However, there have been no studies examining the effects of fasting status on miRNA expression profiles.

Lifestyle may also affect serum miRNA expression. A number of recent studies have focused on the use of circulating miRNAs as lung cancer biomarkers $[10,16,26]$. Because $\sim 85 \%$ of lung cancers in men and $45 \%$ in women are caused by smoking [27], there is a need to examine the effects of smoking on circulating miRNA profiles before these molecules can be used as lung cancer biomarkers. One study has shown that serum levels of miR-625* are significantly lower in non-small cell lung cancer patients who smoke compared to those who do not smoke [28]. However, there have been no studies comparing the serum miRNA levels of healthy smokers to healthy non-smokers. Other unknown factors or lifestyle changes may influence the serum miRNA profiles of healthy individuals leading to a change in these profiles over time.

In this study, we examined the effect of hemolysis, fasting, and smoking on serum miRNA profiles of healthy individuals and compared serum miRNA profiles of samples taken from healthy individuals over different time periods. Our results contribute to a better understanding of the pre-analytical factors that may influence serum miRNA profiles.

\section{Methods}

\section{Ethics statement}

Written consent was obtained from all patients prior to obtaining blood samples. Use of human specimens in this work was approved by the University of British Columbia ethics board (H10-02846).

\section{Sample collection}

To determine the effects of hemolysis on serum miRNA profiles, two vials of blood were drawn from 10 healthy volunteers. One vial was lysed immediately after collection by passing the blood through a 20 gauge needle several times. This method has been shown to mimic mechanical hemolysis due to improper blood collection or specimen preparation [29]. To assess serum miRNA variability in healthy individuals and to determine the effects of fasting on serum miRNA profiles, blood samples were drawn from 7 healthy volunteers one hour after eating a fatty meal and again, three weeks later, after fasting overnight. Serum triglyceride levels were measured for each sample using a Triglyceride Assay Kit (Cayman Chemical Company, Ann Arbor, MI, USA). To determine the effects of smoking on serum miRNA profiles, blood samples were collected from 10 current smokers and from 10 age and sex-matched non-smokers without evidence of disease. Finally, to examine changes in serum miRNA levels over time two samples were collected from 12 healthy volunteers over various time periods. All blood samples were drawn into serum separator vacutainer tubes and kept at room temperature for $30 \mathrm{mi}$ nutes to allow clotting. Clotted samples were centrifuged at room temperature (15 minutes, $1500 \mathrm{rcf}$ ), aliquoted, and frozen at $-80^{\circ} \mathrm{C}$ within 2 hours of collection.

\section{Hemoglobin concentration}

Serum absorbance levels were measured spectrophotometrically using a NanoDrop ND1000 spectrophotometer (NanoDrop Technologies). The hemoglobin concentration of each sample was calculated using the Harboe method [30] with the following formula:

$$
\begin{aligned}
& \operatorname{Hemog} \operatorname{lobin}(\mathrm{g} / \mathrm{l})=\left(167.2 \mathrm{~A}_{415}-83.6 \mathrm{~A}_{380}-83.6 \mathrm{~A}_{450}\right) \\
& \times 1 / 1000
\end{aligned}
$$

where $A_{415}, A_{380}$ and $A_{450}$ are the absorption at 415, 380 and $450 \mathrm{~nm}$ respectively.

\section{RNA purification}

Total RNA was extracted from $200 \mu \mathrm{L}$ of serum using miRNeasy Mini Kit (Qiagen) as previously described [31]. 
For some samples a synthetic spike-in consisting of $20 \mathrm{fmol}$ of synthetic C. elegans miR-39-3p (cel-miR-39-3p; Qiagen, Toronto, ON, Canada) miRNA was added to each sample after a 5 minute incubation in QIAzol Lysis Reagent.

\section{miRNA quantification by qRT-PCR}

The levels of 742 miRNAs (target sequences available at www.exiqon.com/plate-layout-files V2.0) were quantified using miRNome real-time PCR panels (Version V2M, Exiqon Inc., Woburn, MA) as previously described [31]. Due to low RNA yields in serum, the concentration of purified RNA could not be reliably measured, hence fixed volumes of eluted RNA (19.2 $\mu \mathrm{L}$ for 768 reactions) were used for miRNA expression assays as per the manufacturers recommendation. For samples profiled with a synthetic spike-in, $1 \mu \mathrm{l}$ of $\mathrm{LNA}^{\mathrm{TM}}$ control cel-mir-39-3p primer (Exiqon) was added to two empty wells on each panel.

\section{Data analysis}

$\mathrm{Ct}$ values and ROX reference dye normalization were calculated using Viia7 Software (Applied Biosystems). All assays were inspected for distinct melting curves - those with $>1 \mathrm{~T}_{\mathrm{m}}$ and those detected within $5 \mathrm{Ct}$ 's of the negative control $(\mathrm{Ct}>35)$ were excluded from analysis. qRTPCR results were exported to GenEx (MultiD Analyses $\mathrm{AB})$ and normalized using inter-plate calibrators on the miRNA Ready-to-Use panels. There are currently no standard endogenous controls for serum miRNA studies [32]. Therefore we selected an endogenous control suitable for each sample set as described in results. Because less abundant miRNAs showed high variability, miRNAs detected in $<80 \%$ of case or control samples were excluded from analyses. To compare matched samples, a fold-change analysis was used ( $2^{\text {(case } \Delta \mathrm{Ct}-\operatorname{control} \Delta \mathrm{Ct})}, \Delta \mathrm{Ct}=$ Raw endogenous control $\mathrm{Ct}$ - Raw assay $\mathrm{Ct}$ ) and to identify the most significantly deregulated miRNAs a 3-fold change cut-off was applied (selection of fold-change (FC) cut-off explained in results). Average-linkage Pearson correlation hierarchical clustering was calculated and displayed using Genesis software (www.genome.tugraz. at). The Mann-Whitney U test was conducted using Statistica Software (Statsoft ${ }^{\mathrm{TM}}$, Tulsa, OK). All $P$-values were corrected for multiple testing using the Benjamini and Hochberg method.

\section{Results}

\section{Determining the best fold-change (FC) cut-off value}

To determine the appropriate FC cut-off to use when identifying significantly differentially expressed miRNAs via a fold-change analysis, we examined the consistency of our assay by analyzing a technical repeat of our quantification methods. Serum collected from a healthy non-smoker was aliquoted into two separate vials (200 $\mu \mathrm{l}$ each) and two separate RNA purification and miRNA quantification experiments were conducted. The results were normalized to miR-99a-5p and 139-5p and a fold-change analysis was conducted. Of the 157 miRNAs detected in both samples, 48 (31\%) miRNAs showed $\geq 2$-fold difference between the two samples, 14 (9\%) showed a $\geq 3$-fold difference, and 5 (3\%) of miRNAs showed a $\geq 4$-fold difference. Because serum samples contain a relatively low concentration of RNA we anticipated that much of the observed variation was resulting from miRNAs with high $\mathrm{Ct}$ values. Indeed, 38 of the 48 miRNAs that exhibited a $\geq 2$-fold difference had raw $\mathrm{Ct}$ values $>30$ and all 48 miRNAs had a raw $\mathrm{Ct}$ value $>28$. Because the improvement between a 3 -fold and 4-fold FC cut-off was modest in comparison to the improvement between a 2-fold and 3-fold FC cut-off, we decided to consider miRNAs with a $\geq 3$-fold difference as significant in further analyses (Figure 1).

\section{The effects of hemolysis on serum miRNA levels}

To determine the effects of hemolysis on serum miRNA profiles, we measured the serum miRNA profiles of lysed and unlysed serum samples from ten healthy volunteers. Hemoglobin levels of all samples were measured spectrophotometrically, all unlysed samples had a hemoglobin concentration below $0.1 \mathrm{~g} / \mathrm{l}$ and all lysed samples had a hemoglobin concentration above $0.1 \mathrm{~g} / \mathrm{l}$ (Table 1).

Out of the 742 miRNAs profiled 109 miRNAs were detected in all 20 samples, 181 miRNAs were detected in all 10 hemolysed samples, and 116 miRNAs were detected in all 10 non-hemolysed samples. 36 miRNAs were detected in at least 5 of the 10 hemolysed samples at a $\mathrm{Ct}$ below 34 but were not detected in any of the matched nonhemolysed samples (Figure 2).

To determine the best endogenous control to use for normalization we first identified 109 miRNAs that were present at detectable levels in all 20 samples. Because we were only interested in intra-individual comparisons, we then calculated the ratios of the raw $\mathrm{Ct}$ values of each miRNA in matched lysed and unlysed samples using the following formula: $2^{\text {( raw Ct (unlysed) - raw Ct (lysed) }}$. miR-122$5 \mathrm{p}$ and miR-1266-5p were the only miRNAs with ratios < 2 in all samples and had the lowest standard deviations of all miRNAs tested (Additional file 1: Table S1). Because miR-122-5p had the lowest average ratio and has been shown by others to be unaffected by hemolysis [20,21] it was used to normalize prior to fold-change analysis.

A synthetic spike-in (cel-miR-39-3p) was added and measured in 14 of the 20 samples to confirm that sample hemolysis did not significantly affect extraction efficiency. Table 2 shows the raw Ct value of the spiked-in RNA for all lysed and unlysed samples. There was no significant difference between the cel-miR-39-3p Ct values of lysed and unlysed samples.

A fold-change analysis identified 4 miRNAs that were up-regulated by at least 3-fold in all hemolysed samples 


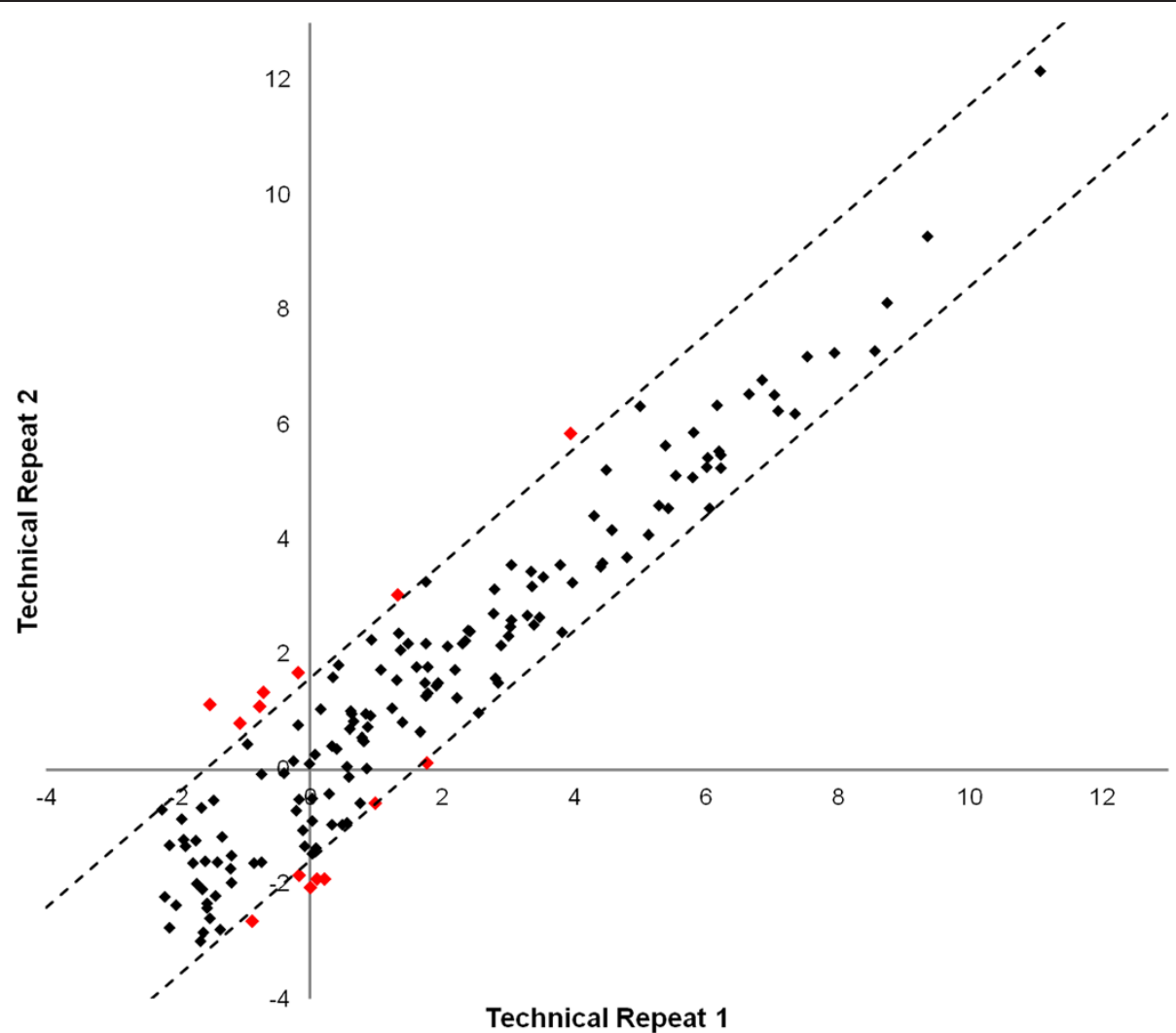

Figure 1 Technical Repeat. A scatter plot of the normalized Ct values for 157 miRNAs from a technical repeat experiment in which serum collected from the same patient was aliquoted and subjected to two separate RNA purification and miRNA quantification steps. The dotted line represents the 3-fold difference boundary between the two samples. The 14 miRNAs showing a $\geq 3$-fold difference are highlighted in red.

and 231 miRNAs that were up-regulated by at least 3fold in at least 1 hemolysed sample compared to unlysed samples. No miRNAs were down-regulated by at least 3fold in more than 1 hemolysed sample. The spike-in was not significantly differentially expressed between hemolysed and non-hemolysed samples suggesting that

\section{Table 1 Hemoglobin concentration}

\begin{tabular}{ccc}
\hline $\begin{array}{c}\text { Sample } \\
\text { ID }\end{array}$ & $\begin{array}{c}\text { Hemoglobin concentration } \\
\text { (g/l) unlysed }\end{array}$ & $\begin{array}{c}\text { Hemoglobin concentration } \\
\text { (g/l) lysed }\end{array}$ \\
\hline 11 & 0.051 & 0.552 \\
12 & 0.048 & 1.217 \\
14 & 0.055 & 0.620 \\
15 & 0.056 & 0.220 \\
16 & 0.042 & 0.154 \\
17 & 0.074 & 1.091 \\
18 & 0.069 & 1.048 \\
19 & 0.075 & 2.428 \\
20 & 0.037 & 1.235 \\
21 & 0.070 & 2.249 \\
\hline
\end{tabular}

Hemoglobin concentrations of matched lysed and unlysed serum samples. the difference in miRNA levels is not due to the presence of inhibitors in the samples. Because clinical samples often contain some hemolysis [33], we have identified a list of miRNAs significantly affected by mechanical hemolysis to consider when conducting serum miRNA biomarker studies. For our purposes, we

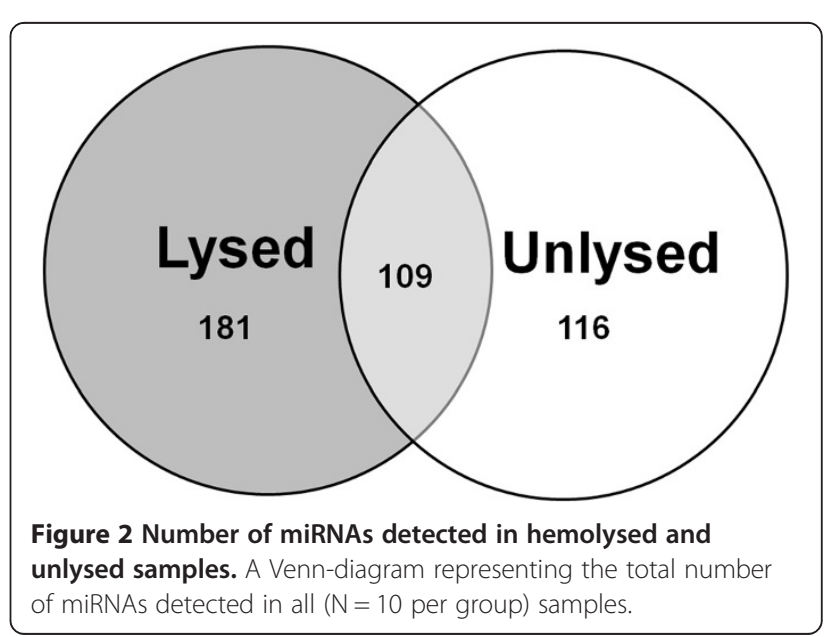


Table 2 Synthetic spike-in levels in hemolysed samples

\begin{tabular}{ccc}
\hline $\begin{array}{c}\text { Sample } \\
\text { ID }\end{array}$ & $\begin{array}{c}\text { cel-miR-39-3p raw } \mathrm{Ct} \\
\text { unlysed sample }\end{array}$ & $\begin{array}{c}\text { cel-miR-39-3p raw Ct } \\
\text { lysed sample }\end{array}$ \\
\hline 15 & 22.70 & 22.20 \\
16 & 22.33 & 22.66 \\
17 & 23.79 & 24.32 \\
18 & 24.01 & 23.46 \\
19 & 23.71 & 22.70 \\
20 & 22.88 & 22.89 \\
21 & 23.75 & 24.04 \\
\hline
\end{tabular}

The raw $\mathrm{Ct}$ values of a synthetic spiked-in RNA (cel-miR-39-3p) for all lysed and unlysed samples demonstrates that hemolysis did not have a significant impact on RNA isolation efficiency.

decided to exclude miRNAs up-regulated by at least 3 -fold in at least $50 \%$ of samples or detected in $50 \%$ of hemolysed samples and not detected in matched unlysed samples leaving a final list of 162 miRNAs that are significantly influenced by hemolysis (Additional file 2 : Table S2).

To measure the correlation between hemoglobin concentration and serum miRNA levels, the raw data was normalized to miR-99a-5p and miR-139-5p (rationale described below). Of the miRNAs detected in at least $80 \%$ of the hemolysed samples or $80 \%$ of the non-hemolysed samples, 177 miRNAs were significantly (corrected $P$-value < 0.05 ) correlated with hemoglobin concentration (Additional file 3: Table S3). A Pearson correlation cluster analysis of miRNAs show that the samples cluster according to hemoglobin concentration and not according to sample source (Figure 3).

\section{Selecting endogenous controls for serum miRNA studies}

Currently, there are no standard endogenous controls for serum miRNA studies [32]. The varying levels of miRNAs observed in samples with high hemoglobin concentrations suggest that some current methods of normalization may be unreliable when samples exhibit hemolysis. Our data suggest that normalizing to liver specific miR-122-5p may be appropriate for matched samples as it is not affected by hemolysis and shows little variation in samples collected from the same person within a short period of time. However, because this miRNA is inconsistently expressed from person to person and has been shown to be deregulated under certain pathologies $[34,35]$ it is not a suitable endogenous control for inter-individual comparisons. To identify a suitable endogenous control for serum miRNA studies, we examined the profiles of 154 serum samples from: 26 lung adenocarcinoma patients, 50 oral CIS/OSCC patients, 18 healthy non-smokers, 43 healthy smokers, and the 7 non-fasting and 10 hemolysed samples described in this study. Of the 46 miRNAs detected in all 154 serum samples, miRs 139-5p and 99a showed the lowest standard deviation across all samples even when the global mean values of these samples were included (Table 3). Therefore, for the following analyses, all data were normalized to the average $\mathrm{Ct}$ values of miR-99a-5p and miR-139-5p.

\section{The effects of fasting on serum miRNA profiles of healthy individuals}

It has been suggested that fasting status may alter serum miRNA profiles due to lipids interfering with the RNA extraction [24] or to miRNAs from food entering circulation [25]. However, to our knowledge, this effect has not yet been tested. To assess the effects of fasting on serum miRNA profiles we collected blood samples from 7 healthy volunteers one hour after eating a fatty meal and again three weeks later, after fasting overnight. Serum triglyceride levels were measured enzymatically and were higher in the non-fasting samples (Table 4). A comparison of the total number of miRNAs detected in each sample showed that, after the exclusion of miRNAs significantly affected by hemolysis, the total miRNA count was slightly higher in the non-fasting samples compared to the fasting samples although not significant $(P=0.27$, Figure 4). After normalization to miR-99a-5p and miR-139-5p, Pearson correlation coefficients between serum triglyceride concentration and miRNA levels showed 6 miRNAs were significantly (4 directly and 2 inversely) correlated with serum triglyceride levels (Table 5). However, a fold-change analysis between matched samples showed that no miRNAs were significantly (by at least 3-fold in at least $50 \%$ of cases) differentially expressed between the fasting and non-fasting samples. No miRNAs were detected in over 3 of the 7 fasting or non-fasting samples at a Ct below 34 and not detected in the corresponding matched sample. A Mann-Whitney $U$ test of miRNAs normalized to miR-99a$5 p$ and miR-139-5p expressed in at least 5 of 7 fasting or 5 of 7 non-fasting samples showed that no miRNAs were significantly $(\mathrm{P}<0.05)$ differentially expressed between the two groups (data not shown).

\section{The effects of smoking on serum miRNA profiles}

To examine the effects of tobacco smoke on serum miRNA expression we compared the profiles of 10 current smokers to 10 age and sex-matched controls (Table 6). A Mann-Whitney U test conducted on miRNAs normalized to miR-99a-5p and miR-139-5p and detected in at least $80 \%$ of smokers or $80 \%$ of non-smokers showed that no miRNAs were significantly (corrected $P$-value $<0.05$ ) differentially expressed. However, miR-128-3p showed the lowest uncorrected $P$-value $(0.001)$ and this miRNA was detected in 7 non-smoker cases and 9 smoker cases and was slightly up-regulated in smokers (Figure 5). No miRNAs were present in only smoking samples or only nonsmoking samples. 
Figure 3 The effect of hemolysis on serum miRNA levels. An average-linkage Pearson correlation cluster analysis of the serum miRNA profiles of matched unlysed (yellow) and lysed (blue) samples collected from 10 healthy individuals. Only miRNAs detected in at least $80 \%$ of lysed samples or $80 \%$ of unlysed samples are included.

\section{Changes in serum miRNA levels over time}

To assess changes in serum miRNA levels in healthy individuals over time we compared the profiles of two samples collected from the same individual over varying time periods (Table 7). Pearson correlation coefficients of the levels of miRNAs detected in at least 19 of 24 samples showed a strong correlation between samples collected over different time periods from the same person (Table 7). A fold-change analysis showed no miRNAs were frequently significantly deregulated between matched samples (up or down-regulated by at least 3-fold in at least $50 \%$ of cases). Nine miRNAs (miR-888-5p, miR-454-3p, miR-10a5p, miR-181c-5p, miR-1909-3p, miR-20a-3p, miR-484, miR-501-5p, and miR-622) were detected in at least 50\% of cases and not detected in the corresponding matched control. Of these nine miRNAs, four (miR-501-5p, miR454-3p, miR-20a-3p, and miR-484) are also in the list of 162 miRNAs significantly affected by hemolysis and therefore the difference in miRNA levels could be due to differences in the amount of hemolysis in each sample.

\section{Discussion}

An ideal biomarker can be measured non-invasively and is highly sensitive and specific to the disease state of interest. The stability of circulating miRNAs and their differential expression in the serum and plasma of patients with cancer and other conditions make these molecules attractive biomarkers. However, in order for a biomarker to have a high positive predictive value it should not be significantly influenced by technical variables or pathologic conditions unrelated to the disease being investigated. In this study we examined the effects of a range of technical and individual factors on serum miRNA levels. Our results have identified a list of miRNAs that are significantly affected by these factors and are therefore likely to have very limited utility as biomarkers of disease.

We have contributed to previous studies examining the role of blood cell contaminants in serum miRNA quantification [19-21]. While Kirschner et al have performed a similar study in plasma [23] and miRNA profiles of blood cells and blood microvesicles have been previously identified [19], to our knowledge, this is the first study specifically examining the effect of mechanical hemolysis on the quantification of 742 miRNAs in matched serum samples. It should be noted that we did not distinguish between microRNAs in total serum and microRNAs in circulating microvesicles (such as exosomes). that Other studies examining the effects of hemolysis on circulating miRNA 
Table 3 Selecting an endogenous control

\begin{tabular}{|c|c|c|c|}
\hline miRNA & Standard deviation* & miRNA & Standard deviation* \\
\hline hsa-miR-139-5p & $3.4 \mathrm{E}-10$ & hsa-miR-18b-5p & 7.6E-09 \\
\hline hsa-miR-99a-5p & 4.4E-10 & hsa-miR-27b-3p & 7.9E-09 \\
\hline hsa-miR-125a-5p & $8.5 \mathrm{E}-10$ & hsa-miR-19a-3p & $8.2 \mathrm{E}-09$ \\
\hline hsa-miR-125b-5p & 1.1. E-09 & hsa-miR-191-5p & $8.2 \mathrm{E}-09$ \\
\hline hsa-let-7d & $1.2 \mathrm{E}-09$ & Global Mean & $9.1 \mathrm{E}-09$ \\
\hline hsa-miR-197-3p & $1.2 \mathrm{E}-09$ & hsa-miR-150-5p & $1.1 \mathrm{E}-08$ \\
\hline hsa-miR-145-5p & $1.5 \mathrm{E}-09$ & hsa-miR-107 & $1.2 \mathrm{E}-08$ \\
\hline hsa-miR-140-5p & 1.6E-09 & hsa-miR-425-5p & 1.4E-08 \\
\hline hsa-miR-342-3p & $1.9 \mathrm{E}-09$ & hsa-miR-27a-3p & $1.5 \mathrm{E}-08$ \\
\hline hsa-miR-215-5p & $1.9 \mathrm{E}-09$ & hsa-miR-142-3p & 1.7E-08 \\
\hline hsa-miR-29a-3p & 2.0E-09 & hsa-miR-140-3p & 2.1E-08 \\
\hline hsa-miR-378a-3p & 2.1E-09 & hsa-miR-122-5p & $2.4 \mathrm{E}-08$ \\
\hline hsa-miR-210-3p & 2.2E-09 & hsa-miR-23a-3p & $2.5 \mathrm{E}-08$ \\
\hline hsa-miR-146a-5p & $2.2 \mathrm{E}-09$ & hsa-miR-103-3p & $2.5 \mathrm{E}-08$ \\
\hline hsa-miR-142-5p & 2.2E-09 & hsa-miR-185-5p & $6.5 \mathrm{E}-08$ \\
\hline hsa-miR-130a-3p & $3.5 \mathrm{E}-09$ & hsa-miR-144-3p & $9.5 \mathrm{E}-08$ \\
\hline hsa-miR-1979 & $3.9 \mathrm{E}-09$ & hsa-miR-106a-5p & $1.2 \mathrm{E}-07$ \\
\hline hsa-miR-23b-3p & 4.7E-09 & hsa-miR-15a-5p & $1.8 \mathrm{E}-07$ \\
\hline hsa-miR-192-5p & 4.9E-09 & hsa-miR-20a-5p & $2.0 \mathrm{E}-07$ \\
\hline hsa-miR-30c-5p & $5.0 \mathrm{E}-09$ & hsa-miR-92a-3p & $3.0 \mathrm{E}-07$ \\
\hline hsa-miR-29c-3p & $5.0 \mathrm{E}-09$ & hsa-miR-223-3p & $3.2 \mathrm{E}-07$ \\
\hline hsa-miR-30b-5p & 5.1E-09 & hsa-miR-16-5p & 9.7E-07 \\
\hline hsa-miR-222-3p & 5.4E-09 & hsa-miR-451a & 4.9E-06 \\
\hline hsa-miR-424-5p & 7.6E-09 & & \\
\hline
\end{tabular}

*All raw $\mathrm{Ct}$ values were converted to a linear scale (using $2^{\wedge}-\mathrm{Ct}$ ) before calculating standard deviation.

The standard deviation of 46 miRNAs detected in all of 154 serum samples. The global mean is included for comparison.

profiles have done so by profiling blood cells directly or by adding hemolysate back into already separated serum or plasma samples [20]. However, Dimeski (2004) demonstrated that samples prepared in this way can have a different effect on analytes than samples prepared by mimicking mechanical hemolysis [29]. By mimicking hemolysis caused by improper blood collection or preparation we have identified a list of 162 miRNAs that are most likely to be

Table 4 Triglyceride concentration in serum

\begin{tabular}{|c|c|c|}
\hline Sample & $\begin{array}{l}\text { Triglyceride concentration } \\
\text { (mg/dl) non-fasting }\end{array}$ & $\begin{array}{l}\text { Triglyceride concentration } \\
\text { (mg/dl) fasting }\end{array}$ \\
\hline 1 & 202.8 & 120.6 \\
\hline 2 & 152.1 & 96.2 \\
\hline 3 & 148.5 & 52.1 \\
\hline 4 & 93.3 & 30.4 \\
\hline 5 & 176.4 & 102.4 \\
\hline 6 & 72.4 & 57.8 \\
\hline 7 & 53.5 & 45.7 \\
\hline
\end{tabular}

Triglyceride concentration of fasting and non-fasting serum samples. affected by hemolysis in a clinical setting. Currently, researchers examining the role of hemolysis on plasma and serum miRNA profiles have suggested excluding hemolysed samples from studies [22]. This method may be useful in identifying biomarkers because it allows the measurement of all miRNAs while avoiding the effects of hemolysis. However, from a clinical standpoint, excluding samples with even low ( $\sim 0.15 \mathrm{~g} / \mathrm{l})$ levels of hemolysis is impractical because the frequency of hemolysis in a clinical setting has been reported as being as high as $5.6 \%$ and samples with low amounts of hemolysis cannot be identified visually [36]. Asking these patients to return for multiple blood draws requires further staff time and supplies and can delay diagnosis leading to increased stress on the patient [37]. Therefore, we propose that rather than excluding hemolysed samples from biomarker studies, researchers should eliminate miRNAs significantly affected by hemolysis when identifying potential biomarkers.

Several groups have addressed the importance of assessing analytical parameters for serum miRNA studies especially with regards to comparing results from multiple 


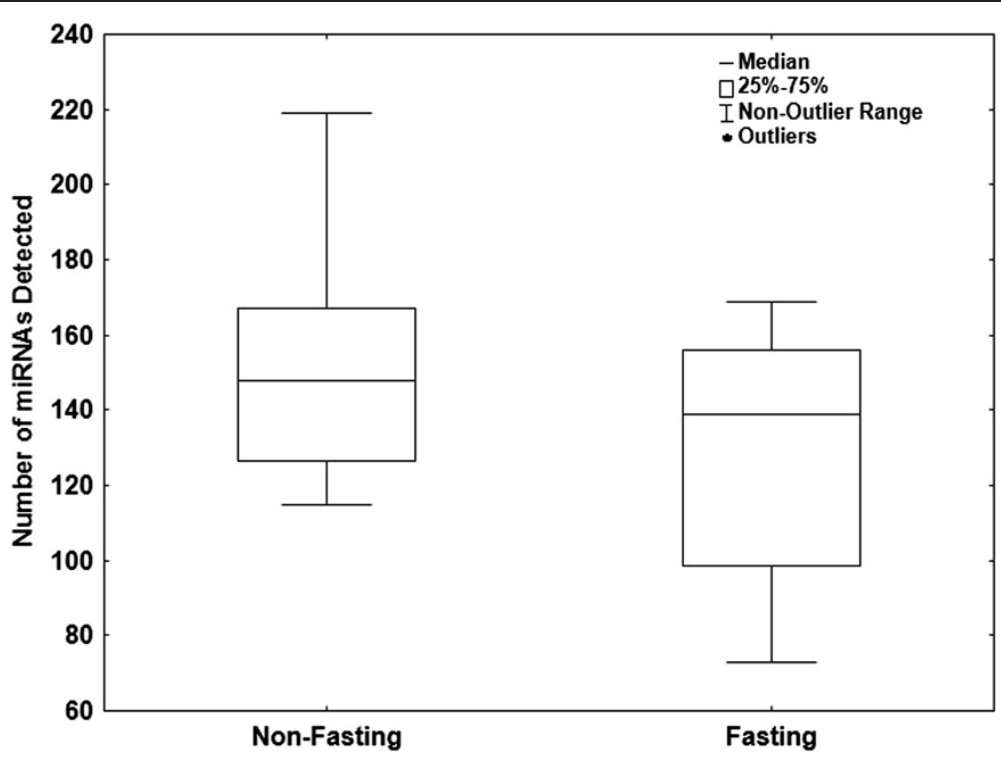

Figure 4 Total number of miRNAs detected in fasting and non-fasting samples. The total number of miRNAs (out of 742 profiled) present at detectable levels in fasting and non-fasting samples. $P$-value $=0.27$, Mann-Whitney $U$ test.

groups [20,38]. In this study we have identified potential endogenous controls that show consistent expression across 154 samples including samples from cancer patients as well as samples exhibiting hemolysis. In cases with samples that may be compromised by hemolysis, this method is more appropriate than some commonly used normalization methods. For example, normalizing to the geometric mean of miRNAs expressed in all samples [39] can produce skewed results if a sample is contaminated with miRNAs from blood cells. This occurs because more highly expressed serum miRNAs, which are used for global mean normalization, are also expressed in blood cells and will be present at higher levels in hemolysed samples [21]. Therefore, samples exhibiting hemolysis will appear to have high RNA input due to an overall lower normalization value and miRNAs that are not expressed in blood cells will seem to be down-regulated in that sample. Another commonly used method for normalization is the use of miR16-5p as an endogenous control [40]. However, we have demonstrated that the levels of serum miR-16-5p are

Table 5 Serum miRNA correlation with triglyceride levels

\begin{tabular}{lcc}
\hline miRNA & Correlation coefficient (R) & P-value \\
\hline hsa-miR-17-3p & 0.88 & $2.9 \mathrm{E}-05$ \\
hsa-miR-375 & 0.70 & 0.005 \\
hsa-miR-328-3p & -0.64 & 0.013 \\
hsa-miR-223-3p & -0.57 & 0.033 \\
hsa-miR-593-3p & 0.57 & 0.034 \\
hsa-miR-130b-5p & 0.55 & 0.040 \\
\hline
\end{tabular}

6 miRNAs significantly $(P<0.05)$ correlated with serum triglyceride levels and corresponding Pearson correlation coefficients. significantly increased in samples with high hemoglobin concentrations suggesting that this method is also inappropriate in cases where some samples may be contaminated with blood cell miRNAs. Finally, another commonly used method for miRNA normalization is the use of U6 as an endogenous control. U6 is a nuclear RNA and has been used to normalize tissue and cell miRNA expression [41]. However, because U6 is localized in the nucleus it should not be present at consistent, high levels in the non-cellular components of blood [32]. Indeed, of the 154 samples analyzed in this study U6 is only present at a detectable level in 84 samples suggesting U6 is not a suitable endogenous control for serum miRNA studies. We propose that miR-99a-5p and miR-139-5p should be used for sample normalization instead of these commonly used endogenous controls due to the fact that they are not significantly affected by hemolysis and that they have the lowest standard deviation across a series of 154 samples (Table 3).

A recent report has shown that plant miRNAs are present in the sera of humans and that these miRNAs are acquired through food intake [25]. This finding

Table 6 Patient demographics

\begin{tabular}{lcc}
\hline & Current-smokers & Non-smokers \\
\hline Males & 3 & 3 \\
Females & 7 & 7 \\
Median age & 60.5 & 52 \\
Age range & $52-67$ & $40-64$ \\
Median pack year & 46.3 & 0 \\
\hline
\end{tabular}

Patient demographics for smoking/non-smoking samples. 


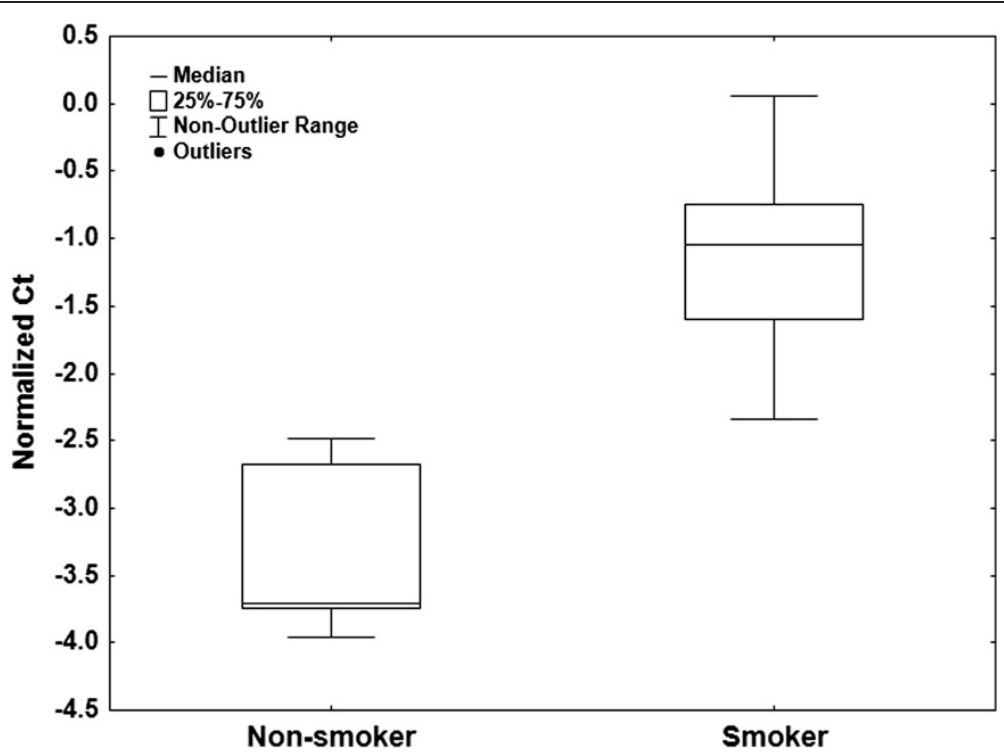

Figure 5 Serum miR-128-3p expression in current smokers and never smokers. Levels of miR-128-3p in the serum of non-smokers and current smokers. Uncorrected $P$-value $=0.004$, Corrected $P$-value $=0.78$, Mann-Whitney $U$ test.

suggests that miRNAs acquired through food intake would show variable expression over time and could be affected by fasting status. Furthermore, fasting status alters the amount of lipids in the blood which could interfere with RNA extraction leading to variable miRNA levels [24]. Here we show that there is no significant difference in the total number of miRNAs detected in fasting versus nonfasting samples demonstrating that the presence of lipids in the blood does not lead to a loss of less abundant miRNAs during extraction. Furthermore, no miRNAs examined in

Table 7 Correlation between samples collected at different time points

\begin{tabular}{ccc}
\hline Sample & Correlation ${ }^{1}$ coefficient & Time between sample collection \\
\hline 1 & 0.88 & 13 months 7 days \\
2 & 0.99 & 15 Months 7 days \\
3 & 0.99 & 17 months 3 days \\
4 & 0.99 & 11 months 9 days \\
5 & 0.98 & 7 months 10 days \\
6 & 0.99 & 5 months 3 days \\
7 & 0.99 & 13 months \\
8 & 0.99 & 12 months 19 days \\
9 & 0.95 & 2 months 14 days \\
10 & 0.99 & 6 months 7 days \\
11 & 0.99 & 10 months \\
12 & 0.87 & 11 months 22 days \\
\hline
\end{tabular}

1. Calculated using miRNAs expressed in at least 19 of 24 samples Pearson correlation coefficients for miRNA profiles of serum collected at two different time points from healthy individuals. our assay were significantly differentially expressed between fasting and non-fasting samples indicating that fasting status will not interfere with serum miRNA biomarker discovery in subjects with normal miRNA physiology. However, 6 miRNAs did show a significant correlation with serum triglyceride levels suggesting some miRNAs may be slightly (but not significantly) influenced by serum lipids. There are limitations to our study including a small sample size and the fact that we only examined short term dietary effects on circulating miRNAs. Therefore, experiments with longterm, controlled diets and large sample sizes need to be carried out in order to rigorously test the overall effects of diet on serum miRNA profiles [42].

As smoking plays an important role in the development of lung and other cancers, it must be considered when identifying potential biomarkers for these diseases. Therefore, we have also examined the influence of smoking on serum miRNA profiles. Because no miRNAs were significantly differentially expressed between smokers and non-smokers our results suggest that smoking status will not interfere with serum miRNA biomarker studies. A recent study using 6-month cigarette smoking in mice found that the levels of miR-128-3p were significantly changed in the lung tissue and plasma of exposed mice suggesting a role for this miRNA in the cellular response to cigarette smoke exposure [43]. We have found that although miR-128-3p was not significantly deregulated in the serum of smokers, this miRNA was detected in more current smoker samples than non-smoker samples and had the lowest P-value prior to correction. Taking the results of both studies into account, the role of smoke 
exposure should be considered when determining the possible utility of miR-128-3p as a biomarker of disease.

When identifying biomarkers for disease detection and/ or monitoring, an important aspect to consider is the possibility of changes in biomarker levels over time in the healthy population. In order to evaluate serum miRNA variability in healthy individuals, we measured the levels of miRNAs in two serum samples taken from 12 individuals over varying time periods. Pearson correlation coefficients of the matched samples show no pattern between correlation coefficient and time between sample collections. This lack of a pattern and the fact that the matched samples with the lowest R-value (0.87) are still highly correlated suggest that, up to 17 months, overall serum miRNA levels show little variability in healthy individuals. Further, longterm, studies are required to determine changes in serum miRNAs over several years. A fold-change analysis showed no individual miRNA was consistently deregulated in these matched samples implying that variability in serum miRNAs over time should not interfere with biomarker studies examining abundant serum miRNAs. Among the less abundant miRNAs, 9 were differentially detected in the matched samples. This result demonstrates that these less abundant miRNAs are not suitable biomarkers due to their inconsistent expression.

\section{Conclusions}

Although this study has limitations such as small sample sizes, the results presented here identify factors that should be taken into consideration when selecting endogenous controls and biomarker candidates. In order for serum miRNA biomarkers to eventually be implemented in a clinical setting, pre-analytical and analytical variables affecting serum miRNA profiles should be examined and standardized. In addition to the variables described in this study other areas requiring further examination include the effect of inflammation, hormonal activity, and diurnal variation on serum miRNA profiles of healthy individuals as well as analytical factors such as the best platform and statistical tests to use for the detection and determination of differentially expressed miRNAs. Once these variables are well defined, the complete potential of circulating miRNAs as biomarkers can be fully explored.

\section{Additional files}

Additional file 1: Table S1. Raw Ct ratios. The ratios of the raw $\mathrm{Ct}$ values of each miRNA in matched lysed and unlysed samples using the following formula: $2 \wedge$ - raw Ct (lysed)/2^-raw Ct (unlysed).

Additional file 2: Table S2. miRNAs significantly affected by hemolysis. A list of miRNAs A) significantly up-regulated (by $\geq 3$-fold) in hemolysed serum samples or B) detected in hemolysed samples at a $\mathrm{Ct} \leq 34$ but not detected in the corresponding matched control.
Additional file 3: Table S3. miRNAs significantly correlated with serum hemoglobin. 177 miRNAs significantly correlated with hemoglobin concentration and corresponding Pearson correlation coefficients.

\section{Competing interests}

The authors declare they have no competing interests.

\section{Authors' contributions}

SAM carried out all experiments and drafted the manuscript. CM and SAM conducted data analyses. SL was responsible for clinical interpretations and sample collection. CG was responsible for overall supervision. All authors contributed equally in study design and have approved the final manuscript.

\section{Acknowledgments}

We would like to thank all the participants in this study for their contribution

Received: 21 October 2013 Accepted: 13 June 2014

Published: 21 June 2014

\section{References}

1. Bartel DP: MicroRNAs: genomics, biogenesis, mechanism, and function. Cell 2004, 116(2):281-297.

2. Lewis BP, Burge CB, Bartel DP: Conserved seed pairing, often flanked by adenosines, indicates that thousands of human genes are microRNA targets. Cell 2005, 120(1):15-20.

3. Brennecke J, Cohen SM: Towards a complete description of the microRNA complement of animal genomes. Genome Biol 2003, 4(9):228.

4. Chen CZ, Li L, Lodish HF, Bartel DP: MicroRNAs modulate hematopoietic lineage differentiation. Science 2004, 303(5654):83-86.

5. Dostie J, Mourelatos Z, Yang M, Sharma A, Dreyfuss G: Numerous microRNPs in neuronal cells containing novel microRNAs. RNA 2003 9(2):180-186.

6. loshikhes I, Roy S, Sen CK: Algorithms for mapping of mRNA targets for microRNA. DNA Cell Biol 2007, 26(4):265-272.

7. Reinhart BJ, Slack FJ, Basson M, Pasquinelli AE, Bettinger JC, Rougvie AE Horvitz HR, Ruvkun G: The 21-nucleotide let-7 RNA regulates developmental timing in Caenorhabditis elegans. Nature 2000, 403(6772):901-906.

8. Xu P, Vernooy SY, Guo M, Hay BA: The Drosophila microRNA Mir-14 suppresses cell death and is required for normal fat metabolism. Curr Biol 2003, 13(9):790-795.

9. Mitchell PS, Parkin RK, Kroh EM, Fritz BR, Wyman SK, Pogosova-Agadjanyan EL, Peterson A, Noteboom J, O'Briant KC, Allen A, Lin DW, Urban N, Drescher CW, Knudsen BS, Stirewalt DL, Gentleman R, Vessella RL, Nelson PS, Martin DB, Tewari M: Circulating microRNAs as stable blood-based markers for cancer detection. Proc Natl Acad Sci U S A 2008, 105(30):10513-10518.

10. Hsu SD, Chu CH, Tsou AP, Chen SJ, Chen HC, Hsu PW, Wong YH, Chen YH, Chen GH, Huang HD: miRNAMap 2.0: genomic maps of microRNAs in metazoan genomes. Nucleic Acids Res 2008, 36(Database issue):D165-D169.

11. Wang K, Zhang S, Marzolf B, Troisch P, Brightman A, Hu Z, Hood LE, Galas DJ: Circulating microRNAs, potential biomarkers for drug-induced liver injury. Proc Natl Acad Sci U S A 2009, 106(11):4402-4407.

12. Ai J, Zhang R, Li Y, Pu J, Lu Y, Jiao J, Li K, Yu B, Li Z, Wang R, Wang L, Li Q, Wang N, Shan H, Li Z, Yang B: Circulating microRNA-1 as a potential novel biomarker for acute myocardial infarction. Biochem Biophys Res Commun 2010, 391(1):73-77.

13. Tsujiura M, Ichikawa D, Komatsu S, Shiozaki A, Takeshita H, Kosuga T, Konishi H, Morimura R, Deguchi K, Fujiwara H, Okamoto K, Otsuji E: Circulating microRNAs in plasma of patients with gastric cancers. Br J Cancer 2010, 102(7):1174-1179.

14. Zhu W, Qin W, Atasoy U, Sauter ER: Circulating microRNAs in breast cancer and healthy subjects. BMC Res Notes 2009, 2:89.

15. Lawrie CH, Gal S, Dunlop HM, Pushkaran B, Liggins AP, Pulford K, Banham AH, Pezzella F, Boultwood J, Wainscoat JS, Hatton CS, Harris AL: Detection of elevated levels of tumour-associated microRNAs in serum of patients with diffuse large B-cell lymphoma. Br J Haematol 2008, 141(5):672-675.

16. Bianchi F, Nicassio F, Marzi M, Belloni E, Dall'olio V, Bernard L, Pelosi G, Maisonneuve P, Veronesi G, Di Fiore PP: A serum circulating miRNA diagnostic test to identify asymptomatic high-risk individuals with early stage lung cancer. EMBO Mol Med 2011, 3(8):495-503. 
17. Brase JC, Wuttig D, Kuner R, Sultmann H: Serum microRNAs as noninvasive biomarkers for cancer. Mol Cancer 2010, 9:306.

18. Hunter MP, Ismail N, Zhang X, Aguda BD, Lee EJ, Yu L, Xiao T, Schafer J, Lee ML, Schmittgen TD, Nana-Sinkam SP, Jarjoura D, Marsh CB: Detection of microRNA expression in human peripheral blood microvesicles. PLoS One 2008, 3(11):e3694.

19. Duttagupta R, Jiang R, Gollub J, Getts RC, Jones KW: Impact of cellular miRNAs on circulating miRNA biomarker signatures. PLoS One 2011, 6(6):e20769

20. McDonald JS, Milosevic D, Reddi HV, Grebe SK, Algeciras-Schimnich A: Analysis of circulating microRNA: preanalytical and analytical challenges. Clin Chem 2011, 57(6):833-840.

21. Pritchard CC, Kroh E, Wood B, Arroyo JD, Dougherty KJ, Miyaji MM, Tait JF, Tewari M: Blood cell origin of circulating microRNAs: a cautionary note for cancer biomarker studies. Cancer Prev Res (Phila) 2012, 5(3):492-497.

22. Blondal T, Jensby Nielsen S, Baker A, Andreasen D, Mouritzen P, Wrang Teilum M, Dahlsveen IK: Assessing sample and miRNA profile quality in serum and plasma or other biofluids. Methods 2012

23. Kirschner MB, Edelman JJ, Kao SC, Vallely MP, van Zandwijk N, Reid G: The Impact of Hemolysis on Cell-Free microRNA Biomarkers. Front Genet 2013, 4:94.

24. Kroh EM, Parkin RK, Mitchell PS, Tewari M: Analysis of circulating microRNA biomarkers in plasma and serum using quantitative reverse transcription-PCR (qRT-PCR). Methods 2010, 50(4):298-301.

25. Zhang L, Hou D, Chen X, Li D, Zhu L, Zhang Y, Li J, Bian Z, Liang X, Cai X, Yin Y, Wang C, Zhang T, Zhu D, Zhang D, Xu J, Chen Q, Ba Y, Liu J, Wang Q, Chen J, Wang J, Wang M, Zhang Q, Zhang J, Zen K, Zhang C: Exogenous plant MIR168a specifically targets mammalian LDLRAP1: evidence of cross-kingdom regulation by microRNA. Cell Res 2012, 22(1):107-126.

26. Yuxia M, Zhennan T, Wei Z: Circulating miR-125b is a novel biomarker for screening non-small-cell lung cancer and predicts poor prognosis. J Cancer Res Clin Oncol 2012, 138(12):2045-2050.

27. Parkin DM, Pisani P, Lopez AD, Masuyer E: At least one in seven cases of cancer is caused by smoking. Global estimates for 1985. Int J Cancer 1994, 59(4):494-504.

28. Roth C, Stuckrath I, Pantel K, Izbicki JR, Tachezy M, Schwarzenbach H: Low levels of cell-free circulating miR-361-3p and miR-625* as blood-based markers for discriminating malignant from benign lung tumors. PLOS One 2012, 7(6):e38248.

29. Dimeski G: Effects of hemolysis on the Roche ammonia method for Hitachi analyzers. Clin Chem 2004, 50(5):976-977.

30. Harboe M: A method for determination of hemoglobin in plasma by near-ultraviolet spectrophotometry. Scand J Clin Lab Invest 1959, 11:66-70.

31. Maclellan SA, Lawson J, Baik J, Guillaud M, Poh CF, Garnis C: Differential expression of miRNAs in the serum of patients with high-risk oral lesions. Cancer Med 2012, 1(2):268-274.

32. Mo MH, Chen L, Fu Y, Wang W, Fu SW: Cell-free Circulating miRNA Biomarkers in Cancer. J Cancer 2012, 3:432-448

33. Lippi G, Salvagno GL, Montagnana M, Brocco G, Guidi GC: Influence of hemolysis on routine clinical chemistry testing. Clin Chem Lab Med 2006, 44(3):311-316.

34. Bihrer V, Friedrich-Rust M, Kronenberger B, Forestier N, Haupenthal J, Shi Y, Peveling-Oberhag J, Radeke HH, Sarrazin C, Herrmann E, Zeuzem S, Waidmann O, Piiper A: Serum miR-122 as a biomarker of necroinflammation in patients with chronic hepatitis C virus infection. Am J Gastroenterol 2011 106(9):1663-1669.

35. Xu J, Wu C, Che X, Wang L, Yu D, Zhang T, Huang L, Li H, Tan W, Wang C, Lin D: Circulating microRNAs, miR-21, miR-122, and miR-223, in patients with hepatocellular carcinoma or chronic hepatitis. Mol Carcinog 2011, 50(2):136-142

36. Lippi G, Salvagno GL, Favaloro EJ, Guidi GC: Survey on the prevalence of hemolytic specimens in an academic hospital according to collection facility: opportunities for quality improvement. Clin Chem Lab Med 2009, 47(5):616-618.

37. Sharp MK, Mohammad SF: Hemolysis in needleless connectors for phlebotomy. ASAIO J 2003, 49(1):128-130.

38. Zampetaki A, Mayr M: Analytical challenges and technical limitations in assessing circulating miRNAs. Thromb Haemost 2012, 108(4):592-598.

39. Mestdagh $P$, Van Vlierberghe P, De Weer A, Muth D, Westermann F, Speleman F, Vandesompele J: A novel and universal method for microRNA RT-qPCR data normalization. Genome Biol 2009, 10(6):R64.
40. Wong TS, Liu XB, Wong BY, Ng RW, Yuen AP, Wei Wl: Mature miR-184 as Potential Oncogenic microRNA of Squamous Cell Carcinoma of Tongue. Clin Cancer Res 2008, 14(9):2588-2592.

41. Zhan M, Miller CP, Papayannopoulou T, Stamatoyannopoulos G, Song CZ: MicroRNA expression dynamics during murine and human erythroid differentiation. Exp Hematol 2007, 35(7):1015-1025.

42. Witwer KW: XenomiRs and miRNA homeostasis in health and disease: evidence that diet and dietary miRNAs directly and indirectly influence circulating miRNA profiles. RNA Biol 2012, 9(9):1147-1154.

43. Huang Y, Dai Y, Zhang J, Wang C, Li D, Cheng J, Lu Y, Ma K, Tan L, Xue F, Qin B: Circulating microRNAs as potential biomarkers for smokingrelated interstitial fibrosis. Biomarkers 2012, 17(5):435-440.

doi:10.1186/1472-6890-14-27

Cite this article as: MacLellan et al:: Pre-profiling factors influencing serum microRNA levels. BMC Clinical Pathology 2014 14:27.

\section{Submit your next manuscript to BioMed Central and take full advantage of:}

- Convenient online submission

- Thorough peer review

- No space constraints or color figure charges

- Immediate publication on acceptance

- Inclusion in PubMed, CAS, Scopus and Google Scholar

- Research which is freely available for redistribution

Submit your manuscript at www.biomedcentral.com/submit
C Biomed Central 